\title{
PAX6 regulates human corneal epithelium cell identity
}

Koji Kitazawa $^{a, b, c}$, Takafusa Hikichia ${ }^{a}$, Takahiro Nakamura ${ }^{b}$, Chie Sotozono $^{c}$, Shigeru Kinoshita $^{\mathrm{b}, *}$ and Shinji Masui ${ }^{\mathrm{a}, \mathrm{d}, *}$

${ }^{1}$ Center for iPS Cell Research and Application, Kyoto University, 53 Kawahara-cho, Shogoin, Sakyo-ku, Kyoto 606-8507, Japan.

${ }^{2}$ Department of Frontier Medical Science and Technology for Ophthalmology, Kyoto Prefectural University of Medicine, 465 Kajii-cho, Hirokoji-agaru, Kawaramachi-dori, Kamigyo-ku, Kyoto 602-0841, Japan

${ }^{3}$ Department of Ophthalmology, Kyoto Prefectural University of Medicine, Kyoto, Japan.

${ }^{4}$ CREST (Core Research for Evolutional Science and Technology), JST (Japan

Science and Technology Agency) 


\begin{abstract}
PAX6, a paired box transcription factor, is necessary for eye development. However, how it regulates the cell identity of human corneal epithelial cells (CECs) is not well understood. We aimed to clarify the function of PAX6 in human CECs using gene knockout via the clustered regularly interspaced short palindromic repeats (CRISPR) and CRISPR associated protein 9 (Cas9) system. We designed guide RNAs for different targets in PAX6. PAX6-depleted CECs maintained the epithelial morphology, but became larger. Global analyses using microarray revealed that down-regulated genes were primarily CEC-specific and included keratin 12, keratin 3, clusterin (CLU), aldehyde dehydrogenase 3 family member A1 (ALDH3A1), angiopoietin-like 7 (ANGPTL7) and transketolase (TKT), while up-regulated genes were primarily epidermis-related and included keratin 10, keratin 1, involucrin (IVL), filaggrin (FLG). These findings suggest that PAX6 maintains CEC identity by regulating differentiation.
\end{abstract}




\section{Introduction}

The cornea is a transparent and well-aligned tissue structure located at the front of the eye and is necessary for good visual function. Its outermost surface is covered with corneal epithelial cells (CECs), which are characterized as non-keratinized stratified epithelial cells that express the corneal specific differentiation markers K3/K12. In severe ocular surface diseases or trauma, such as Stevens-Johnson syndrome, chemical burns and ocular cicatricial pemphigoid, corneal epithelial stem cells around the limbal area disappear. The loss of these cells is associated with superficial neovascularization from the peripheral cornea, subcorneal opacity and symblepharon on the ocular surface, among other pathologies(Sotozono et al., 2007). Corneal and conjunctival epithelia at the chronic stage of such diseases show abnormal differentiation toward pathologic keratinization, leading to the disruption of homeostasis on the ocular surface and ultimately visual disorder(Nakamura et al., 2002; Nakamura et al., 2001; Nishida et al., 1999).

To date, several genes have been reported to maintain CEC identify(Kinoshita et al., 2001). For example, mutations in genes for keratin 3 (KRT3) and keratin 12 (KRT12) are associated with Meesmann corneal dystrophy, which is characterized by fragility of the corneal epithelium and the presence of small cysts(Nishida et al., 1997). Another gene, aldehyde dehydrogenase 3 family member A1 (ALDH3A1), reportedly protects CECs from oxidative stress(Estey et al., 2007). We recently revealed that the transduction of PAX6 with several other transcription factors directly induces human CEC-like cells that express KRT3, KRT12 and ALDH3A1 from skin fibroblasts(Kitazawa et al., 2016). Furthermore, we previously reported that clusterin (CLU) was the most abundant gene in the human corneal epithelium and is essential 
for maintaining the ocular surface epithelium as non-keratinized(Nakamura et al., 2002; Nishida et al., 1998).

To investigate how PAX6 regulates the gene expression profile of CECs, we considered knocking down PAX6 expression. In general, gene knockdown can be done with RNA interference (RNAi). In RNAi, however, residual gene products normally remain and off-target effects can lead to unexpected gene expression changes, which sometimes results in unwanted toxicities and obfuscates interpretation of the phenotypic changes(Jackson and Linsley, 2010). The clustered regularly interspaced short palindromic repeats (CRISPR) and CRISPR associated protein 9 (Cas9) system is a relatively new genome editing technology that is remarkably easy to use and accurate(Cho et al., 2013; Cong et al., 2013; Jinek et al., 2012; Mali et al., 2013). Although it too can suffer from off-target effects, recent developments have minimized this risk(Kleinstiver et al., 2016; Shen et al., 2014). To completely abolish PAX6 expression in CECs, we designed two guide RNAs so that Cas9 would target specific genomic loci to make a double-strand break that results in a nonsense mutation upon non-homologous end joining (NHEJ). We found that PAX6 knockout via the CRISPR/Cas9 system resulted in a loss of CEC identity through the downregulation of corneal epithelial-related genes and the up-regulation of epidermis- and keratinization-related genes.

\section{Materials and methods}

\subsection{Cell culture}

Human normal corneal tissues were obtained from donors from Seattle Eye Bank (Seattle, WA), and human CECs were isolated from these tissues as described previously(Kitazawa et al., 2016). Briefly, CECs were isolated using dispase (Dispase 
type II; Godo Shusei) and TrypLE Express (Life Technologies Corp., Carlsbad, CA), and cultured in CEC medium, which consisted of Dulbecco's modified Eagle's medium and Ham's F-12 media (1:1 mixture) (Life Technologies Corp.) containing B27 supplement (Life Technologies Corp.), Rho-kinase (ROCK) inhibitor (10 $\mu \mathrm{M})$ (Nacalai Tesque Inc., Kyoto, Japan), epidermal growth factor (10 ng/ml) (Life Technologies Corp.), Epigallocatechin-gallate (SIGMA-ALDRICH, St. Louis, MO) and penicillin-streptomycin $(50 \mathrm{IU} / \mathrm{ml})$ (Nacalai Tesque Inc.) at $37{ }^{\circ} \mathrm{C}$ in a $5 \% \mathrm{CO}_{2}$ incubator. Primary CECs from donors were seeded on a single well in a 6-well plate coated with $0.1 \%$-gelatin and were cultured expansively until subconfluence. After one passage, primary CECs were used for the following experiments.

\subsection{Design of target site by CRISPR/Cas9}

We used lentiCRISPR (a gift from Feng Zhang (Addgene plasmid \# 52961)(Sanjana et al., 2014)), which is a lentivirus vector carrying guide RNAs targeted for CRISPR and Cas9, to perform the functional analysis of transcription factors. All sgRNAs, where each sgRNA targeted a particular DNA sequence that included a 23 mer nucleotide and a PAM sequence ( $N N G$, were designed using the website: tool.genome-engineering.org to eliminate the most likely off-target sites. Furthermore, to avoid off-target effects, the two independent guide RNAs, which targeted different sites, were designed in the paired box domain, which is a necessary domain in the PAX6 gene for PAX6 function. Each pair of oligos was annealed and sub-cloned into the lentiCRISPR plasmid by digestion with BsmBI (NEB). The lentiCRISPR plasmid also carried the puromycin resistant gene. Guide RNAs for different targets in PAX6 and EGFP were: sgRNA-PAX6-1: TTCGGGAAATGTCGCACGGC, sgRNA-PAX6- 
GAGCTGGACGGCGACGTAAA,

\section{CAAGTTCAGCGTGTCCGGCG.}

\subsection{Lentivirus preparation}

Lentivirus was freshly prepared according to a previous report, but with minor modifications(Kitazawa et al., 2013). Briefly, 293T cells were seeded at a density of $4.0 \times 10^{5}$ cells onto a single well of a 6 -well plate coated with $0.1 \%$ gelatin in $2 \mathrm{ml}$ of DMEM (Nacalai Tesque) with 10\% FBS. $7.2 \mu$ of Lipofectamine 2000 (Life Technologies Corp.) was added to $100 \mu$ l of OPTIMEM (Life Technologies Corp.), and the mixture was incubated at room temperature for 5 minutes. After that, $0.8 \mu \mathrm{g}$ of lentiCRISPR with the targeted guide RNA, $0.8 \mu \mathrm{g}$ of package plasmid psPAX2 (Addgene \#12260) and $0.8 \mu \mathrm{g}$ of envelope plasmid pCMV-VSVG diluted in $100 \mu 1$ of OPTIMEM were added to the mixture and incubated at room temperature for 20 minutes. 24 hours after the transduction, the culture medium was changed, and after another 24 hours the supernatant was harvested and added to a cell culture.

\subsection{Retrovirus preparation}

Retrovirus was prepared according to a previous report(Hikichi et al., 2013). Plat-GP cells were seeded at a density of $4.0 \times 10^{5}$ cells onto a single well of a 6 -well plate coated with $0.1 \%$-gelatin in $2 \mathrm{ml}$ of DMEM (Nacalai Tesque) and 10\% FBS.

FuGENE 6 transfection reagent (Promega) was diluted with $60 \mu \mathrm{l}$ of OPTIMEM, and the mixture was incubated at room temperature for 5 minutes. $1.2 \mu \mathrm{g}$ of pMXs-EGFP vector DNA and $0.6 \mu \mathrm{g}$ of VSV-G envelope vector were added to the mixture. The mixture was then incubated at room temperature for another 20 minutes. After incubation, the mixture was added onto Plat-GP cells. The next day, the culture 
medium was changed with the same volume, and the virus-containing supernatant was harvested after another 24 hours.

\subsection{Gene knockout}

The constructed plasmid was transfected into 293 T cells. 48 hours after the transfection, $250 \mu 1$ of the supernatant was harvested and transferred to primary CECs. For lentiviral transduction, the virus-containing supernatant was added to the cultures of the dissociated CECs in the presence of $5 \mu \mathrm{g} / \mathrm{ml}$ polybrene. The cells were transfected using the spin infection method. Briefly, the cell plate was set up in a centrifuge at $35^{\circ} \mathrm{C}$, and the cells were centrifuged for 30 minutes at $800 \times \mathrm{g}$. After washing, the medium was replaced with CEC culture medium. In the EGFP-knockout experiments, primary CECs were seeded at a density of $2.0 \times 10^{4}$ cells onto a single well of a 12-well plate, and then pMXs retrovirus carrying EGFP with neomycin resistant gene was infected into primary CECs. 7 days after infection and selection with $300 \mathrm{ng} / \mu \mathrm{l}$ neomycin, $100 \%$ of the CECs expressed EGFP, and lentiCRISPRcarrying guide RNAs targeting EGFP were transduced in EGFP-expressing CECs. In PAX6-knockout experiments, lentiCRISPR-carrying guide RNAs targeting PAX6 were transduced in primary CECs at a density of $2.0 \times 10^{4}$ cells onto a single well of a 12-well plate. After transduction of lentiCRISPR followed by selection with $1 \mu \mathrm{g} / \mathrm{ml}$ puromycin, primary CECs were harvested at day 7 and then assessed by qRT-PCR and western blotting. In general, primary CECs without transduction of the lentiCRISPR plasmid were dead within 2 days after puromycin selection. The lentiCRISPR without guide RNA (i.e. empty vector) was used as the control. Cell size was calculated by analyzing cell counts and area with Image J $1.48 \mathrm{v}$ software. 


\subsection{Immunohistochemistry}

Normal human cornea was cryopreserved until the time of use. Immunohistochemistry was performed according to a previously reported method with modifications(Kitazawa et al., 2013). Briefly, the tissues were dried, and Zamboni solution (phosphate-buffered combination of picric acid and paraformaldehyde) or Acetone was added at $4{ }^{\circ} \mathrm{C}$ for 10 minutes to fix the samples. After washing, the samples were permeabilized with $0.05 \%$ Triton-X-100 at room temperature for 30 minutes. After that, the samples were blocked with $2 \%$ BSA, incubated overnight at $4{ }^{\circ} \mathrm{C}$ with PAX6 antibody (Santa Cruz; AD2.35, mouse), keratin (K) 12 antibody (Santa Cruz; N-16, goat)、K3 antibody (PROGEN; AE-5, mouse), K10 antibody (Leica Biosystems; LHP1, mouse) and involucrin (IVL) (Leica Biosystems; SY5, mouse) diluted in 2\% BSA. The samples were washed again with PBS and incubated at room temperature for 1 hour with the appropriate secondary antibody diluted in 2\% BSA. Finally, propidium iodide (PI) or 4',6-diamidino-2phenylindole (DAPI) was added, and the signal was detected using a fluorescence microscope (AX70 TRF; Olympus Corp., Tokyo, Japan).

\subsection{Microarray}

Microarray was performed as previously described with modifications(Hikichi et al., 2013). Total RNA was extracted using RNeasy kit (QIAGEN, Hilden, Germany) and assessed using Agilent 2100 Bioanalyzer and RNA 6000 LabChip kit (Agilent Technologies Inc., Santa Clara, CA). High quality RNA was used in the subsequent experiments. Microarray experiments were performed according to the manufacturer's instructions. 100 ng of total RNA was labelled with Cyanine 3-CTP and hybridized to Human Gene 2.0 ST Array (Affymetrix Inc., Santa Clara, CA). The 
array data were analyzed using GeneSpring software ver.13.0 (Agilent Technologies Inc.). Gene expression values were normalized following the exclusion of low-signal intensity data and percentile shifts. To analyze the global expression changes in primary CECs with empty vector or PAX6-depleted CECs, probes showing more than 2.0-fold up-regulation or down-regulation were used for the consequent analyses. The biological functions of the genes were analyzed using DAVID functional annotation analysis (http://david.abcc.ncifcrf.gov), and the expression levels between control and PAX6-depleted CECs of corneal-epithelial related genes and epidermis-related genes were compared.

\subsection{Quantitative reverse transcription polymerase chain reaction (qRT-PCR)}

Complementary DNA was generated from $1 \mu \mathrm{g}$ RNA by reverse transcription with random hexamer primer and oligo dT primer using commercial reverse transcriptase (Superscript $^{\circledR}$ III First-Strand Synthesis SuperMix for qRT-PCR; Life Technologies Corp.). Each PCR primer for the genes of interest was designed using free online primer design tool (Primer 3: http://bioinfo.ut.ee/primer3-0.4.0/). A reaction mixture with cDNA, primer and GoTaq ${ }^{\circledR}$ Green Master Mix (Promega Corp., Fitchburg, WI) was run on a StepOne instrument (Applied Biosciences, Waltham, MA). The primer pairs are listed in Table 1.

\subsection{Western Blotting Analysis}

Western blotting analysis was performed as previously described(Kitazawa et al., 2013). Briefly, proteins were resolved on a 4-20\% gradient SDS-polyacrylamide gel (Mini-PROTEAN ${ }^{\circledR}$ TGX ${ }^{\mathrm{TM}}$; Bio-Rad Laboratories Inc., Hercules, CA). The protein extracts were transferred to a PVDF membrane (Trans-Blot ${ }^{\circledR}$ Turbo ${ }^{\mathrm{TM}}$ Transfer Pack; 
Bio-Rad Laboratories Inc.), which was then blocked in TBS-T (Tris-buffered saline with $0.05 \%$ Tween 20 ) buffer containing $1 \%$ skim milk incubated overnight at $4{ }^{\circ} \mathrm{C}$ with the primary antibodies anti-PAX6 (COVANCE; PRB-278P, rabbit), anti-HistonH3 (Cell Signaling, HRP-conjugate), anti-K3 (PROGEN; AE-5, mouse), anti-K12 (abcam; ab124975, rabbit) anti-K10 (abcam; ab76318, rabbit) and anti-IVL (Leica Biosystems; SY5, mouse). After washing three times, the membrane was incubated with an HRP-conjugated secondary antibody at room temperature for 1 hour and washed again. A chemiluminescent reagent (ECL Advance Western Blotting Detection Kit; GE Healthcare, Little Chalfont, UK) was then applied onto the blotted membrane. The luminescent signal was detected using a chilled charge-coupled device (CCD) digital imaging camera (LAS-3000UVmini; Fujifilm Corp., Tokyo, Japan).

\subsection{Statistical Analysis}

Statistical analyses were performed using statistical software (JMP®pro ver. 12.1.0). Comparisons between groups were conducted with Student's t-test. P values less than 0.05 were considered significant.

\section{Results}

\subsection{Characteristics of human corneal epithelium}

Corneal epithelium is stratified epithelium and consists of 5 to 6 layers. We tested the

distribution of PAX6 in normal central human cornea. Immunohistochemistry demonstrated that PAX6 was expressed in CECs throughout all layers (Fig. 1A). The distribution of K12 and K3 was also throughout all layers (Figs. 1B and 1C). K10, which is an epidermis-related gene, was not expressed in the corneal epithelium (Fig. 
1D), and IVL, which is a keratinized-related gene, was observed in only the superficial layer (Fig. 1E).

\subsection{Design of sgRNA for EGFP and PAX6}

To address the function of PAX6 in primary CECs, we used the CRISPR/Cas9 system to make a double-strand break. Breaks at the target site are usually repaired by the NHEJ DNA repair pathway. This mechanism usually results in a loss of a few to several hundred nucleotides around the cleavage site, although insertions are sometimes observed, resulting in the loss of protein expression (Fig. 2A). We examined whether lentiCRISPR-carrying guide RNAs targeting EGFP or PAX6 can knockout these genes in human primary CECs. We designed sgRNAs for EGFP and PAX6 at the earliest constitutive exon (Figs. 2B and 2C). To reduce off-target effects and clarify gene function, we designed guide RNA for two different target sites and used www.cripsr.tool.//, which provides data on all off-target sites for the predicted targets and evaluates the mismatch rate 20 mers within the candidate sgRNA sequences we designed.

\subsection{Depletion of EGFP and PAX6 using CRISPR/Cas9 system}

We transduced lentiCRISPR that carried the designed sgRNAs to human CECs. LentiCRISPR without sgRNAs was used as a control. Culture protocol of EGFP depletion in primary CEC was shown in Fig. 3A. Before the infection of lentiCRISPR, EGFP was transduced in primary CECs via pMXs retrovirus carrying EGFP, and the

CECs were cultured with neomycin selection. Beginning 2 days after the lentiCRISPR infection, the EGFP expression level gradually decreased with puromycin treatment during the culture. Finally at day 9, lentiCRISPR for EGFP 
abolished the EGFP protein, while control cells did not show reduced EGFP expression (Fig. 3B). Next, we used lentiCRISPR for PAX6 in primary CECs. PAX6depleted CECs exhibited an epithelial morphology similar to that of control. However, the size of these cells seemed larger (Figs. 3C and 3D). PAX6 protein was abolished in CECs transduced with two different lentiCRISPR-carrying sgRNAs (Fig. 3E).

3.4. Global expression analysis in human corneal epithelial cells lacking PAX6 Next, global analyses between PAX6-depleted CECs and CECs with empty vector (control) using microarray were performed. We found that 760 genes were differentially expressed more than two-fold between the two cell types, with 371 genes up-regulated and 389 genes down-regulated (Fig. 4A and Supplemental Table 1). DAVID functional annotation analysis revealed that the up-regulated genes were significantly linked to keratinocyte $\left(p=5.77 \times 10^{-7}, 11\right.$ transcripts $)$ and skin $(p=$ 0.044, 26 transcripts), while the down-regulated genes were significantly linked to cornea ( $\mathrm{p}=0.0051,3$ transcripts) (Figure 4B and Supplemental Tables 2 and 3). The up-regulated genes were enriched to epidermis- and keratinized-related genes including KRT10 and IVL, while the down-regulated genes were enriched to CECrelated genes including KRT3 and KRT12. In addition, CLU, ALDH3A1, ANGPTL7, and lumican (LUM), which have different functions in CECs, were also downregulated (Fig. 4C).

3.5. PAX6 regulated corneal epithelial-related genes and epidermis-related genes Next, to validate the results of the microarray analysis, we performed qRT-PCR and western blot analysis. The qRT-PCR analysis found that PAX6 depletion led to significantly decreased expressions of KRT12, KRT3, CLU, ANGPTL7, ALDH3A1 
and transketolase (TKT), which are all corneal epithelial-related genes, but increased the expression of KRT1, KRT10, IVL and filaggrin (FLG), which are all epidermisrelated genes (Fig. 5A). Western blot analysis revealed that the loss of PAX6 upregulated K10 and IVL and down-regulated K12 and K3 at the protein level (Fig. 5B). These findings suggest that PAX6 regulates genes related to CEC terminal differentiation and neovascularization via the inhibition of epidermal differentiationrelated genes and determines the gene profile of CECs. 


\section{Discussion}

In this study, PAX6 function in primary human CECs was for the first time examined via its abolishment by the CRISPR/Cas9 system. We found that depletion of PAX6 in primary CECs down-regulated KRT3 and KRT12, but up-regulated KRT10 and IVL, demonstrating the loss of CEC identity. This result suggests that PAX6 regulates the gene expression profile of CECs and is necessary for CECs to take non-keratinized epithelial cell properties.

The CRISPR/Cas9 system was ideal for this study, because it permits doublestranded breaks at specific spots in the DNA sequence. Although some indel mutations are expected to maintain the reading frame, homozygous knockout yields high screening sensitivity(Shalem et al., 2014), which is especially important in cases where incomplete knockdown retains gene function. As a comparison, we also used siRNA to knockdown PAX6, but found inferior reduction of PAX6 and smaller changes in the expression of target genes (Supplemental Fig. 1). The deletion of PAX6 by CRISPR/Cas9 with two independent and different target guide RNAs resulted in similar phenotypic changes.

Our work demonstrated that human CECs absent PAX6 exhibit a down-regulation of corneal epithelial-related genes and up-regulation of epidermis-related genes (Figs. $4 \mathrm{~B}, 5 \mathrm{~A}$ and $5 \mathrm{~B})$. This result is consistent with a recent report that found the transduction of PAX6 in skin epithelial stem cells is sufficient to convert them to corneal epithelial stem cell-like cells(Ouyang et al., 2014). The same research group showed that the loss of PAX6 converts corneal epithelial stem cells to epidermal stem cells(Li et al., 2015). Another report showed that the down-regulation of Pax6 is associated with the abnormal differentiation of non-keratinized cells to keratinized cells in severe ocular surface diseases(Li et al., 2008). We previously reported that 
CLU is important to maintain the ocular surface epithelium as nonkeratinized(Nakamura et al., 2002; Nishida et al., 1998), suggesting that PAX6 regulates the CLU expression level and prevents abnormal differentiation. At the protein level, K12 and K3 expression still remained, but only slightly, in PAX6depleted CECs. One possible explanation for the residual expression is that we did the analysis at day 7 after infection and the complete phenotypic changes of CECs might take more time. We think that a longer CECs culture method would help explain how PAX6 regulates $\mathrm{K} 12$ and $\mathrm{K} 3$ in more detail.

At the developmental stage, the boundary between PAX6-expressing and nonexpressing cells is precisely defined at the edge of the eyelid, with PAX6-expressing cells located on the interior surface of the eyelid where they develop into ocular surface cells, but they are not found on the outside of the eyelid where cells differentiate into epidermal cells(Grindley et al., 1995; Koroma et al., 1997). It has been reported that the transplantation of mouse embryonic stem cells (ESCs) overexpressing PAX6 on the ocular surface restore corneal epithelial function(Ueno et al., 2007). These findings indicate that PAX6 is an important transcription factor for lineage segregation between ocular surface cell fate and epidermal cell fate. Microarray analyses revealed the down-regulation of not only differentiated markers, but also CLU, which is a non-keratinizing marker(Nakamura et al., 2002), and Angiopoietin-Like 7 (ANGPTL7), which reportedly prevents neovascularization on the ocular surface(Toyono et al., 2015), indicating that PAX6 may contribute to corneal avascularity. In addition, promoter analysis revealed that PAX6 directly binds to the promoter of KRT12(Shiraishi et al., 1998) and to ALDH3A1, activating its gene expression(Davis et al., 2008). Pax $6^{+-}$mice show thinner corneal epithelium and reduced K12 expression in the corneal epithelium, resulting in epithelial fragility and 
epithelial defects(Davis et al., 2003; Ramaesh et al., 2003). These findings suggest that PAX6 maintains CEC identity in terms of corneal function, such as barrier function and avascularity.

However, there is much evidence that PAX6 is necessary for not only the eye but also the central nervous system and pancreas(Ashery-Padan et al., 2004; Walther and Gruss, 1991; Zhang et al., 2010). Zhang and associates demonstrated that the overexpression of PAX6 in human ESCs could directly convert neuroectoderm cells. CECs are the same lineage, surface ectoderm, as epidermal cells, and the two cell types share similar gene expression profiles. Therefore, we propose that the gene expression profiles of CECs and epidermal cells is regulated by PAX6.

In summary, the results of this study demonstrate for the first time that depletion of PAX6 in CECs via the CRISPR/Cas9 system leads to significantly stronger phenotypic changes than does PAX6 knockdown via siRNA. Based on our findings, we conclude that PAX6 regulates genes related to normal differentiation in the cornea by suppressing epidermis-related genes. It is expected that these new findings will lead to better understanding of the gene regulation required for the establishment and maintenance of the corneal epithelium.

\section{Acknowledgements}

We are grateful to Akitsu Hotta, Hongmei Lisa Li and Masahiro Nakamura for scientific discussion, and Takuya Yamamoto and Toshiko Sato for technical support of the mircroarray experiments. We also thank Feng Zhang for providing lentivirus plasmids and Peter Karagiannis for reading the manuscript. This work was supported in part by CREST, JST (to S.M.), The Japanese Society for the Promotion of Science (JSPS) KAKENHI Grant Number 24615004 (to S.M.) and Grant Number 16K20324 
(to K.K.), Core Center for iPS Cell Research, Research Center Network for

Realization of Regenerative Medicine from Japan Agency for Medical Research and

Development, AMED (to S.M.), iPS Cell Research Fund (to S.M), The Funding

Program for World-Leading Innovative Research and Development on Science and

Technology (FIRST Program) of JSPS (to S.M.) and KAKENHI Grant Number

23390404 (to S.K.). This work was also supported by research funds from the Kyoto

Foundation for the Promotion of Medical Science. 


\section{References}

Ashery-Padan, R., Zhou, X., Marquardt, T., Herrera, P., Toube, L., Berry, A., Gruss, P., 2004. Conditional inactivation of Pax6 in the pancreas causes early onset of diabetes. Developmental biology 269, 479-488.

Cho, S.W., Kim, S., Kim, J.M., Kim, J.S., 2013. Targeted genome engineering in human cells with the Cas9 RNA-guided endonuclease. Nature biotechnology 31, 230 232.

Cong, L., Ran, F.A., Cox, D., Lin, S., Barretto, R., Habib, N., Hsu, P.D., Wu, X., Jiang, W., Marraffini, L.A., Zhang, F., 2013. Multiplex genome engineering using CRISPR/Cas systems. Science 339, 819-823.

Davis, J., Davis, D., Norman, B., Piatigorsky, J., 2008. Gene expression of the mouse corneal crystallin Aldh3a1: activation by Pax6, Oct1, and p300. Invest Ophthalmol Vis Sci 49, 1814-1826.

Davis, J., Duncan, M.K., Robison, W.G., Jr., Piatigorsky, J., 2003. Requirement for Pax6 in corneal morphogenesis: a role in adhesion. Journal of cell science 116, 2157 2167.

Estey, T., Piatigorsky, J., Lassen, N., Vasiliou, V., 2007. ALDH3A1: a corneal crystallin with diverse functions. Exp Eye Res 84, 3-12.

Grindley, J.C., Davidson, D.R., Hill, R.E., 1995. The role of Pax-6 in eye and nasal development. Development 121, 1433-1442.

Hikichi, T., Matoba, R., Ikeda, T., Watanabe, A., Yamamoto, T., Yoshitake, S., Tamura-Nakano, M., Kimura, T., Kamon, M., Shimura, M., Kawakami, K., Okuda, A., Okochi, H., Inoue, T., Suzuki, A., Masui, S., 2013. Transcription factors interfering with dedifferentiation induce cell type-specific transcriptional profiles. 
Proceedings of the National Academy of Sciences of the United States of America $110,6412-6417$.

Jackson, A.L., Linsley, P.S., 2010. Recognizing and avoiding siRNA off-target effects for target identification and therapeutic application. Nature reviews. Drug discovery 9 , $57-67$.

Jinek, M., Chylinski, K., Fonfara, I., Hauer, M., Doudna, J.A., Charpentier, E., 2012. A programmable dual-RNA-guided DNA endonuclease in adaptive bacterial immunity. Science 337, 816-821.

Kinoshita, S., Adachi, W., Sotozono, C., Nishida, K., Yokoi, N., Quantock, A.J., Okubo, K., 2001. Characteristics of the human ocular surface epithelium. Progress in retinal and eye research 20,639-673.

Kitazawa, K., Hikichi, T., Nakamura, T., Mitsunaga, K., Tanaka, A., Nakamura, M., Yamakawa, T., Furukawa, S., Takasaka, M., Goshima, N., Watanabe, A., Okita, K., Kawasaki, S., Ueno, M., Kinoshita, S., Masui, S., 2016. OVOL2 Maintains the Transcriptional Program of Human Corneal Epithelium by Suppressing Epithelial-toMesenchymal Transition. Cell reports.

Kitazawa, K., Kawasaki, S., Shinomiya, K., Aoi, K., Matsuda, A., Funaki, T., Yamasaki, K., Nakatsukasa, M., Ebihara, N., Murakami, A., Hamuro, J., Kinoshita, S., 2013. Establishment of a human corneal epithelial cell line lacking the functional TACSTD2 gene as an in vitro model for gelatinous drop-like dystrophy. Invest Ophthalmol Vis Sci 54, 5701-5711.

Kleinstiver, B.P., Pattanayak, V., Prew, M.S., Tsai, S.Q., Nguyen, N.T., Zheng, Z., Joung, J.K., 2016. High-fidelity CRISPR-Cas9 nucleases with no detectable genomewide off-target effects. Nature 529, 490-495. 
Koroma, B.M., Yang, J.M., Sundin, O.H., 1997. The Pax-6 homeobox gene is expressed throughout the corneal and conjunctival epithelia. Invest Ophthalmol Vis Sci 38, 108-120.

Li, G., Xu, F., Zhu, J., Krawczyk, M., Zhang, Y., Yuan, J., Patel, S., Wang, Y., Lin, Y., Zhang, M., Cai, H., Chen, D., Zhang, M., Cao, G., Yeh, E., Lin, D., Su, Q., Li, W.W., Sen, G.L., Afshari, N., Chen, S., Maas, R.L., Fu, X.D., Zhang, K., Liu, Y., Ouyang, H., 2015. Transcription Factor PAX6 (Paired Box 6) Controls Limbal Stem Cell Lineage in Development and Disease. The Journal of biological chemistry 290, 20448-20454.

Li, W., Chen, Y.T., Hayashida, Y., Blanco, G., Kheirkah, A., He, H., Chen, S.Y., Liu, C.Y., Tseng, S.C., 2008. Down-regulation of Pax6 is associated with abnormal differentiation of corneal epithelial cells in severe ocular surface diseases. The Journal of pathology 214, 114-122.

Mali, P., Yang, L., Esvelt, K.M., Aach, J., Guell, M., DiCarlo, J.E., Norville, J.E., Church, G.M., 2013. RNA-guided human genome engineering via Cas9. Science 339, $823-826$

Nakamura, T., Nishida, K., Dota, A., Kinoshita, S., 2002. Changes in conjunctival clusterin expression in severe ocular surface disease. Invest Ophthalmol Vis Sci 43, $1702-1707$.

Nakamura, T., Nishida, K., Dota, A., Matsuki, M., Yamanishi, K., Kinoshita, S., 2001. Elevated expression of transglutaminase 1 and keratinization-related proteins in conjunctiva in severe ocular surface disease. Invest Ophthalmol Vis Sci 42, 549-556. Nishida, K., Honma, Y., Dota, A., Kawasaki, S., Adachi, W., Nakamura, T., Quantock, A.J., Hosotani, H., Yamamoto, S., Okada, M., Shimomura, Y., Kinoshita, S., 1997. Isolation and chromosomal localization of a cornea-specific human keratin 
12 gene and detection of four mutations in Meesmann corneal epithelial dystrophy. American journal of human genetics 61, 1268-1275.

Nishida, K., Kawasaki, S., Kinoshita, S., 1998. Clusterin may be essential for maintaining ocular surface epithelium as a non-keratinizing epithelium. Advances in experimental medicine and biology 438, 629-635.

Nishida, K., Yamanishi, K., Yamada, K., Dota, A., Kawasaki, S., Quantock, A.J., Kinoshita, S., 1999. Epithelial Hyperproliferation and Transglutaminase 1 Gene Expression in Stevens-Johnson Syndrome Conjunctiva. The American Journal of Pathology 154, 331-336.

Ouyang, H., Xue, Y., Lin, Y., Zhang, X., Xi, L., Patel, S., Cai, H., Luo, J., Zhang, M., Zhang, M., Yang, Y., Li, G., Li, H., Jiang, W., Yeh, E., Lin, J., Pei, M., Zhu, J., Cao, G., Zhang, L., Yu, B., Chen, S., Fu, X.D., Liu, Y., Zhang, K., 2014. WNT7A and PAX6 define corneal epithelium homeostasis and pathogenesis. Nature 511, 358-361. Ramaesh, T., Collinson, J.M., Ramaesh, K., Kaufman, M.H., West, J.D., Dhillon, B., 2003. Corneal abnormalities in Pax6+/- small eye mice mimic human aniridia-related keratopathy. Invest Ophthalmol Vis Sci 44, 1871-1878.

Sanjana, N.E., Shalem, O., Zhang, F., 2014. Improved vectors and genome-wide libraries for CRISPR screening. Nature methods 11, 783-784.

Shalem, O., Sanjana, N.E., Hartenian, E., Shi, X., Scott, D.A., Mikkelsen, T.S., Heckl, D., Ebert, B.L., Root, D.E., Doench, J.G., Zhang, F., 2014. Genome-scale CRISPRCas9 knockout screening in human cells. Science 343, 84-87.

Shen, B., Zhang, W., Zhang, J., Zhou, J., Wang, J., Chen, L., Wang, L., Hodgkins, A., Iyer, V., Huang, X., Skarnes, W.C., 2014. Efficient genome modification by CRISPRCas9 nickase with minimal off-target effects. Nature methods 11, 399-402. 
Shiraishi, A., Converse, R.L., Liu, C.Y., Zhou, F., Kao, C.W., Kao, W.W., 1998.

Identification of the cornea-specific keratin 12 promoter by in vivo particle-mediated gene transfer. Invest Ophthalmol Vis Sci 39, 2554-2561.

Sotozono, C., Ang, L.P., Koizumi, N., Higashihara, H., Ueta, M., Inatomi, T., Yokoi, N., Kaido, M., Dogru, M., Shimazaki, J., Tsubota, K., Yamada, M., Kinoshita, S., 2007. New grading system for the evaluation of chronic ocular manifestations in patients with Stevens-Johnson syndrome. Ophthalmology 114, 1294-1302.

Toyono, T., Usui, T., Yokoo, S., Taketani, Y., Nakagawa, S., Kuroda, M., Yamagami, S., Amano, S., 2015. Angiopoietin-like 7 is an anti-angiogenic protein required to prevent vascularization of the cornea. PloS one 10, e0116838.

Ueno, H., Kurokawa, M.S., Kayama, M., Homma, R., Kumagai, Y., Masuda, C., Takada, E., Tsubota, K., Ueno, S., Suzuki, N., 2007. Experimental transplantation of corneal epithelium-like cells induced by Pax6 gene transfection of mouse embryonic stem cells. Cornea 26, 1220-1227.

Walther, C., Gruss, P., 1991. Pax-6, a murine paired box gene, is expressed in the developing CNS. Development 113, 1435-1449.

Zhang, X., Huang, C.T., Chen, J., Pankratz, M.T., Xi, J., Li, J., Yang, Y., Lavaute, T.M., Li, X.J., Ayala, M., Bondarenko, G.I., Du, Z.W., Jin, Y., Golos, T.G., Zhang, S.C., 2010. Pax6 is a human neuroectoderm cell fate determinant. Cell stem cell 7, 90-100. 


\section{Author Contributions}

K.K., S.M. and S.K.: conception and design; K.K. and T.H.: collection and/or assembly of data; K.K., T.H., T.N., C.S., S.K. and S.M.: data analysis and interpretation; K.K., T.N., and S.M.: writing manuscript. All authors reviewed the manuscript. 


\section{Figure Legends}

Fig. 1. Immunohistochemistry of human central corneal epithelium. Human corneal epithelium was stained positive for PAX6 (A), keratin (K) 3 (B) and K12 (C), negative for K10 (D) and partially positive for involucrin (E). Scale bars, $50 \mu \mathrm{m}$.

Fig. 2. The clustered regularly interspaced short palindromic repeats (CRISPR) and CRISPR associated protein 9 (Cas9) system. A: Scheme of knockout via the CRISPR/Cas9 system. B: Guide RNAs for EGFP. Red characters are the sequence of sgRNA_1 and sgRNA_2. C: Guide RNAs for PAX6. The PAX6 cDNA is represented as a horizontal box. The boundary between exons is shown by vertical black lines. 5a indicates an alternatively spliced exon. The sequences of sgRNA_1 and sgRNA_2 are within the paired box region. PB: paired box, LNK: linker region, HB, homeobox, PST: proline/serine/threonine-rich domain.

Fig. 3. Depletion of EGFP and PAX6 in primary human corneal epithelial cells (CECs). A: Culture protocol of EGFP depletion in primary CECs. B: Morphology and EGFP expression in CECs at day 0 and day 9. Scale bars, $200 \mu \mathrm{m}$ (upper images) and $500 \mu \mathrm{m}$ (lower images). C: Morphology of PAX6-depleted CECs and CECs with empty vector (control). Scale bars, $20 \mu \mathrm{m}$. D: Average cell size of PAX6-depleted CECs and control $($ all $n=4)$. Data were analyzed using the Student $t$-test and are shown as means \pm s.d. ${ }^{*} P<0.05$. E: Western blotting analysis of PAX6 protein in PAX6-depleted CECs and control.

Fig. 4. Global analyses of PAX6-depleted human corneal epithelial cells (CECs). A: Scatter plots analysis between PAX6-depleted CECs and CECs with empty vector 
(control) in up-regulated genes (probe 371) and down-regulated genes (probe 389). B:

DAVID functional annotation analysis shows UP genes were enriched keratinocyteor epidermis-related genes (left panel) and DOWN genes were enriched cornearelated genes (right panel). C: Gene expression profile of corneal-related genes and epidermis-related genes in PAX6-depleted CECs compared to control.

Fig. 5. Validation of gene expression changes in PAX6-depleted human corneal epithelial cells. A: Quantitative RT-PCR showed down-regulation of CEC-related genes (upper panel) and up-regulation of epidermis-related genes (lower panel). Data were analyzed using the Student $t$-test and are shown as means \pm s.d. ${ }^{*} P<0.01, \mathrm{n}=$ 4. B: Western blotting analysis of keratin 12 , keratin 3, keratin 10 and involucrin in PAX6-depleted CECs and CECs with empty vector (control). KRT12: keratin 12, KRT3: keratin 3, CLU: clusterin, ALDH3A1: aldehyde dehydrogenase 3 family member A1, ANGPTL7: angiopoietin-like 7, TKT: transketolase, KRT10: keratin 10, IVL: involucrin, KRT1: keratin 1, FLG: filaggrin. 
Table 1

List of oligomers used for this study.

\begin{tabular}{ll}
\hline Primer & Sequence \\
\hline KRT12_forward & 5'-ACTTCAATCCCTATTTTTGTCTGTTC-3' \\
KRT12_reverse & 5'-CTACTCCAGGTCCAGAAGGATATAAG -3' \\
KRT3_forward & 5'-AGGAGCGGGAACAGATCAAGA-3' \\
KRT3_reverse & 5'-GGAACCGCACCTTGTCAATG -3' \\
KRT10_forward & 5'-TCCTACTTGGACAAAGTTCGGG-3' \\
KRT10_reverse & 5'-CCCCTGATGTGAGTTGCCA-3' \\
KRT1_forward & 5'-CCCAGTACGAGGATATAGCCC-3' \\
KRT1_reverse & 5'-GATCACACGATTCAGCTCAGAA-3' \\
CLU_forward & 5'-ACGGTGGCTTCCCACACTT-3' \\
CLU_reverse & 5'-TTCACGACCACCTCAGTGACA-3' \\
ALDH3A1_forward & 5'-TGTTCTCCAGCAACGACAAGG-3' \\
ALDH3A1_reverse & 5'-AGGGCAGAGAGTGCAAGGT-3' \\
ANGPTL7_forward & 5'-CGGCTGCGTGTAGAGATGGA-3' \\
ANGPTL7_reverse & 5'-CCTTGGTGCTGAAGGCTGTGT-3' \\
TKT_forward & 5'-CTGCTTCATCCGGACCAG-3' \\
TKT_reverse & 5'-CACACTTCATACCCGCCCTA \\
IVL_forward & 5'-GCAGGACGGACAACTAAAACA-3' \\
IVL_reverse & 5'-GCTGGTTGAATGTCTTGGACC-3' \\
FLG_forward & 5'-GCACTCGTCATGCAGAGACTT-3' \\
FLG_reverse & 5'-GACCCTCGGTTTCCACTGT-3' \\
PAX6_forward & 5'-TGGGCAGGTATTACGAGACTG-3' \\
PAX6_reverse & 5'-ACTCCCGCTTATACTGGGCTA-3' \\
GAPDH_forward & 5'-GAAGGTGAAGGTCGGAGT-3' \\
GAPDH_reverse & 5'-GAAGATGGTGATGGGATTTC-3' \\
\hline
\end{tabular}


Figure 1
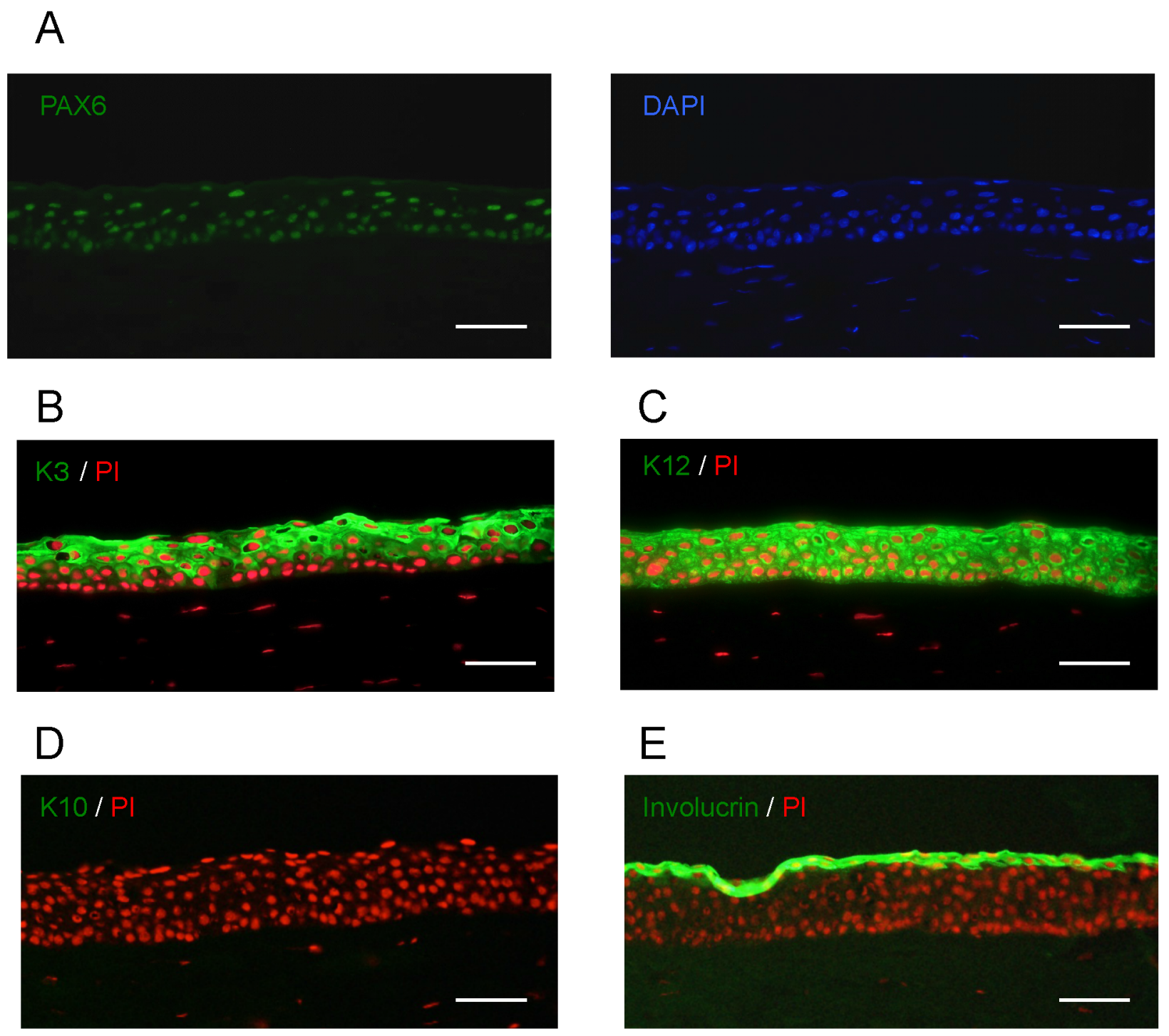


\section{Figure 2}

A

B

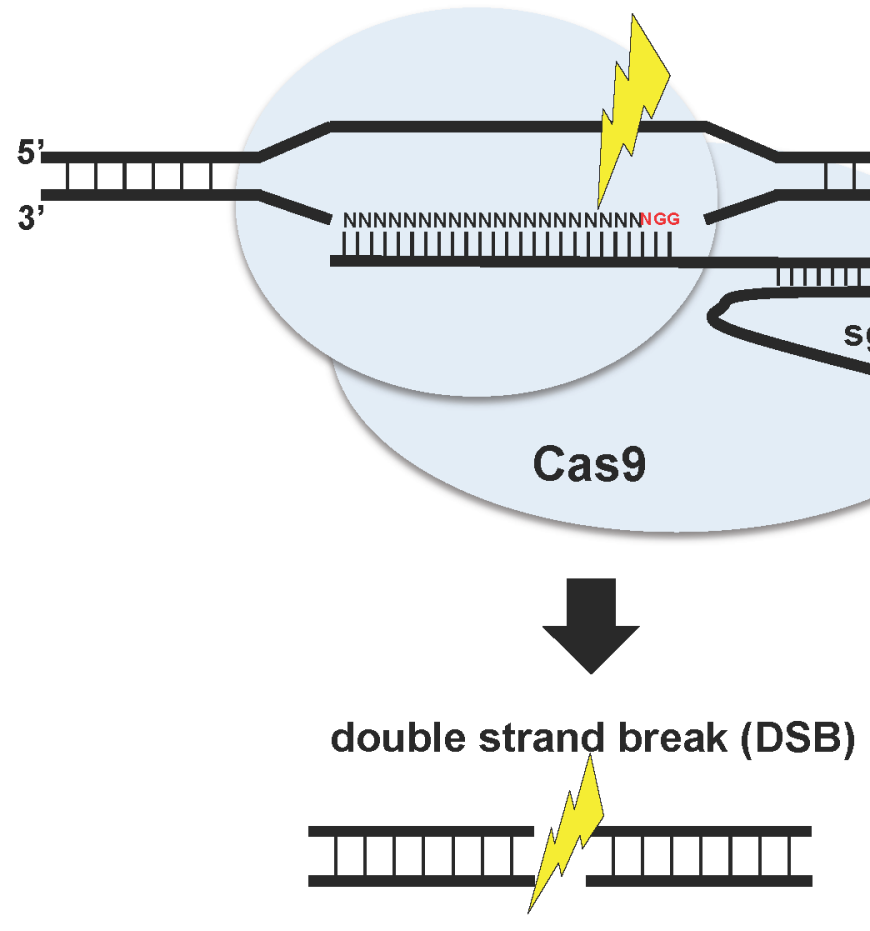

non-homologous end joining (NHEJ)

\section{Insertions/deletions}

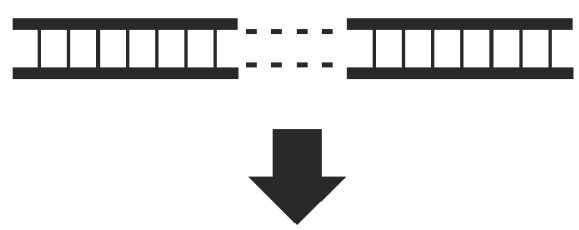

ATGGTGAGCAAGGGCGAGGAGCTGTTCACCGGGGTGGTGCCC ATCCTGGTCGAGCTGGACGGCGACGTAAACGGCCACAAGTTCA GCGTGTCCGGCGAGGGCGAGGGCGATGCCACCTACGGCAAGC TGACCCTGAAGTTCATCTGCACCACCGGCAAGCTGCCCGTGCC CTGGCCCACCCTCGTGACCACCCTGACCTACGGCGTGCAGTGC TTCAGCCGCTACCCCGACCACATGAAGCAGCACGACTTCTTCAA GTCCGCCATGCCCGAAGGCTACGTCCAGGAGCGCACCATCTTC TTCAAGGACGACGGCAACTACAAGACCCGCGCCGAGGTGAAGT TCGAGGGCGACACCCTGGTGAACCGCATCGAGCTGAAGGGCAT CGACTTCAAGGAGGACGGCAACATCCTGGGGCACAAGCTGGAG TACAACTACAACAGCCACAACGTCTATATCATGGCCGACAAGCAG AAGAACGGCATCAAGGTGAACTTCAAGATCCGCCACAACATCGA GGACGGCAGCGTGCAGCTCGCCGACCACTACCAGCAGAACACC CCCATCGGCGACGGCCCCGTGCTGCTGCCCGACAACCACTACC TGAGCACCCAGTCCGCCCTGAGCAAAGACCCCAACGAGAAGCG CGATCACATGGTCCTGCTGGAGTTCGTGACCGCCGCCGGGATC

ACTCTCGGCATGGACGAGCTGTACAAGTAA
sqRNA

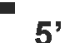

年
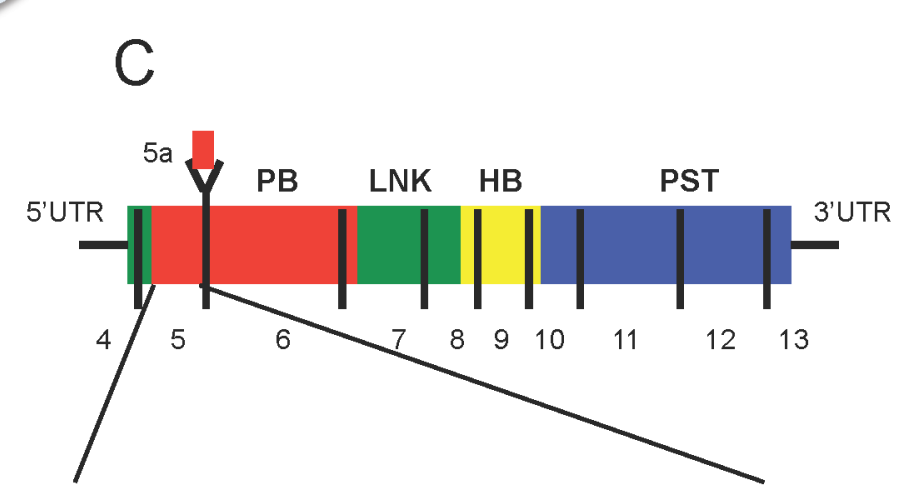

GTCACAGCGGAGTGAATCAGCTCGGTGGTGTC sgRNA_1

TTIGTCAACGGGCGGCCACTGCCGGACTCCAC

CCGGCAGAAGATTGTAGAGCTAGCTCACAGCG sgRNA_2 GGGCCCGGCCGTGCGACATTTCCCGAATTCTG CAG

\section{Gene knockout}




\section{Figure 3}

A

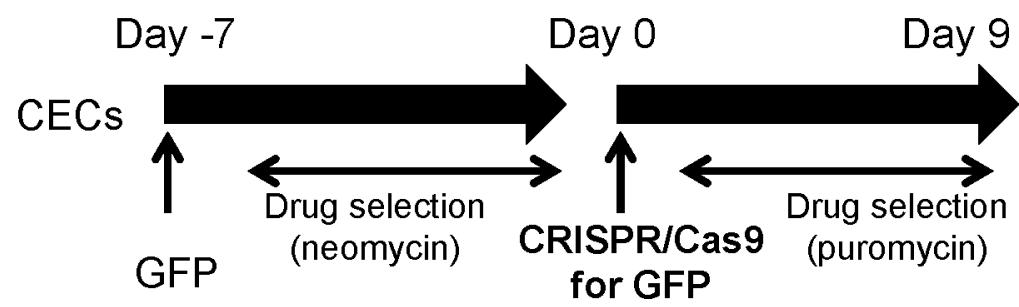

C

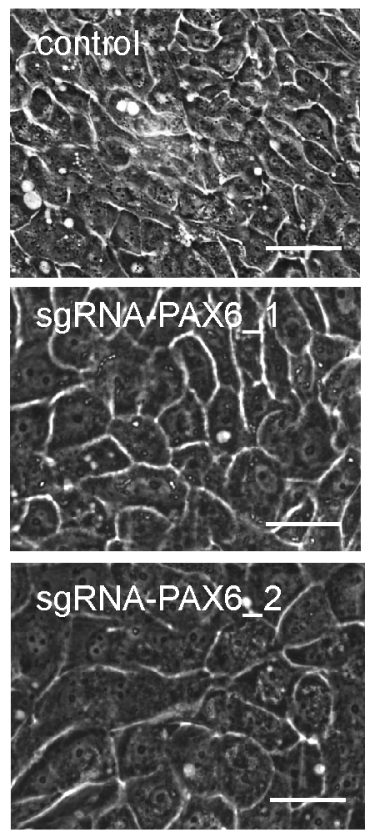

B

Day 0

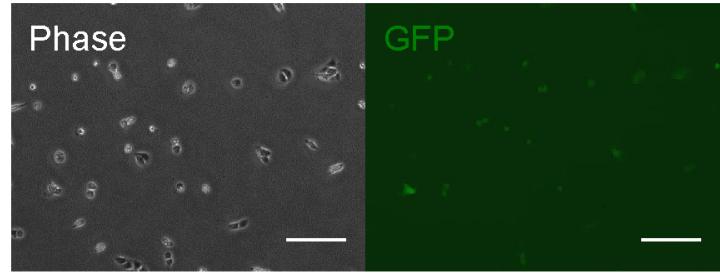

Day 9

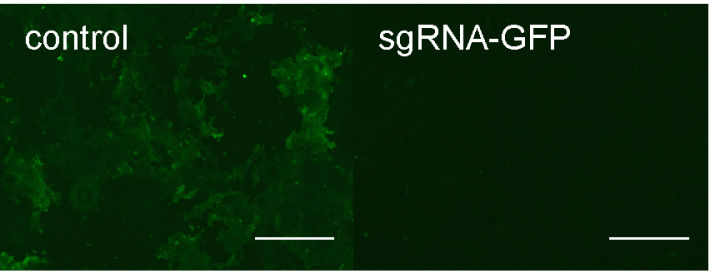

E

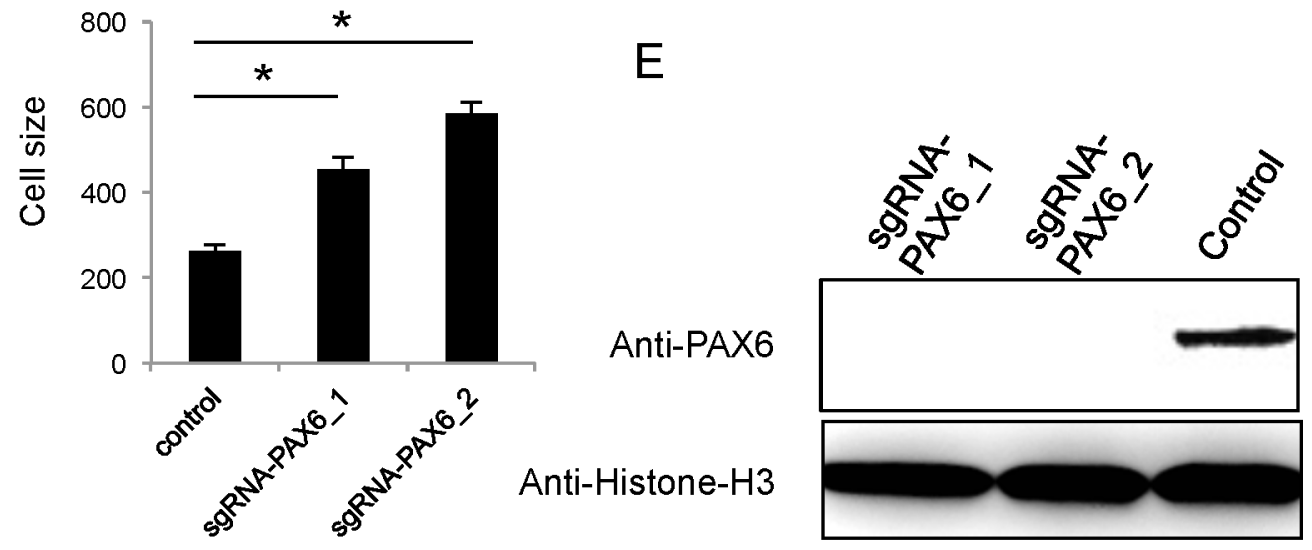




\section{Figure 4}

A

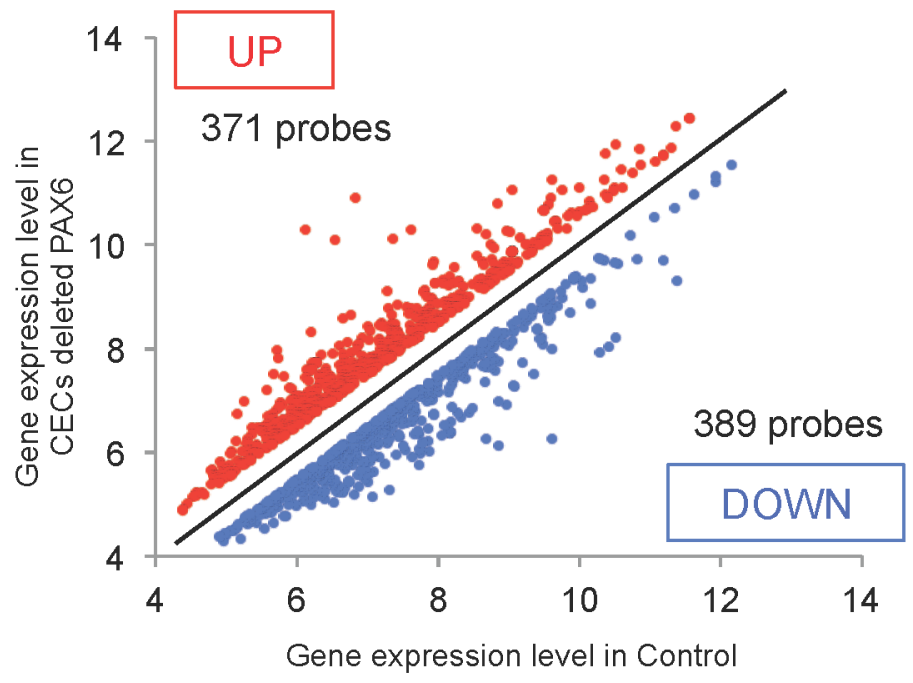

C

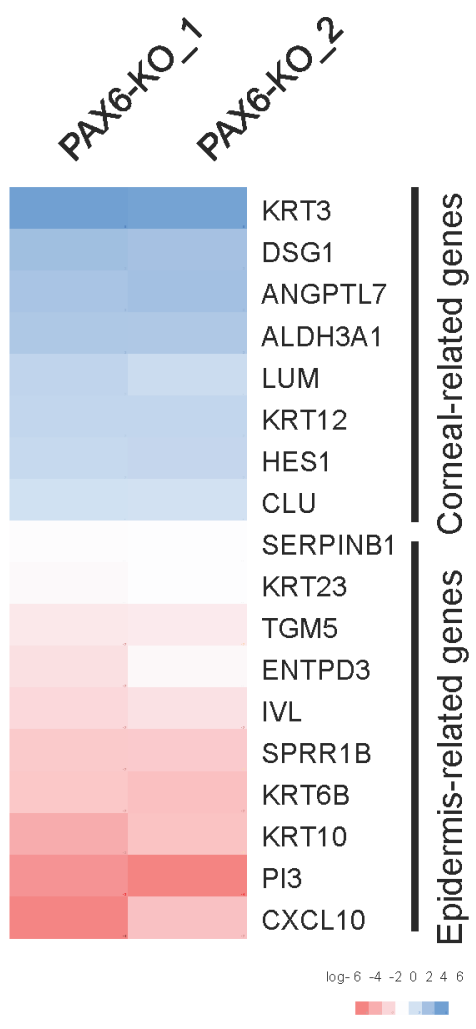

B

\section{UP genes}
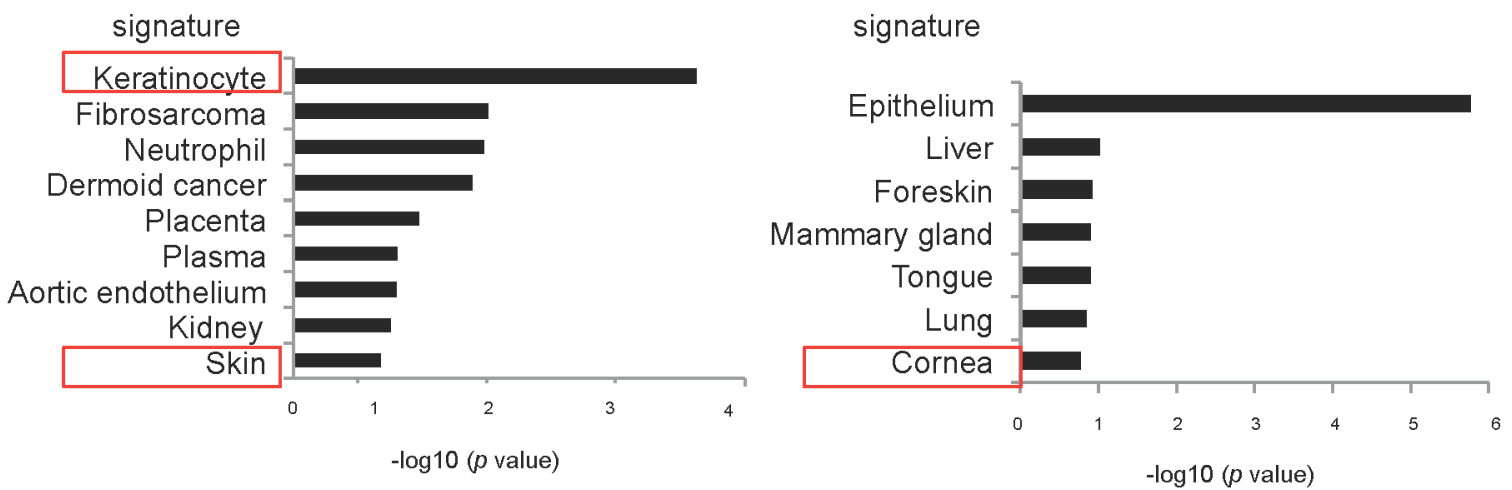


\section{Figure 5}

A

KRT12

KRT3

CLU

ALDH3A1 ANGPTL7

TKT
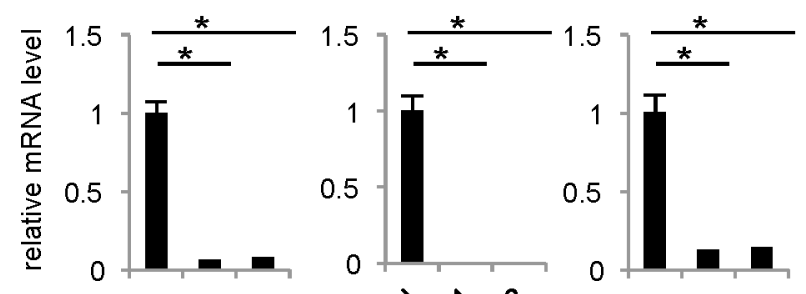

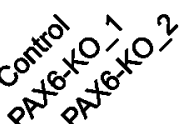
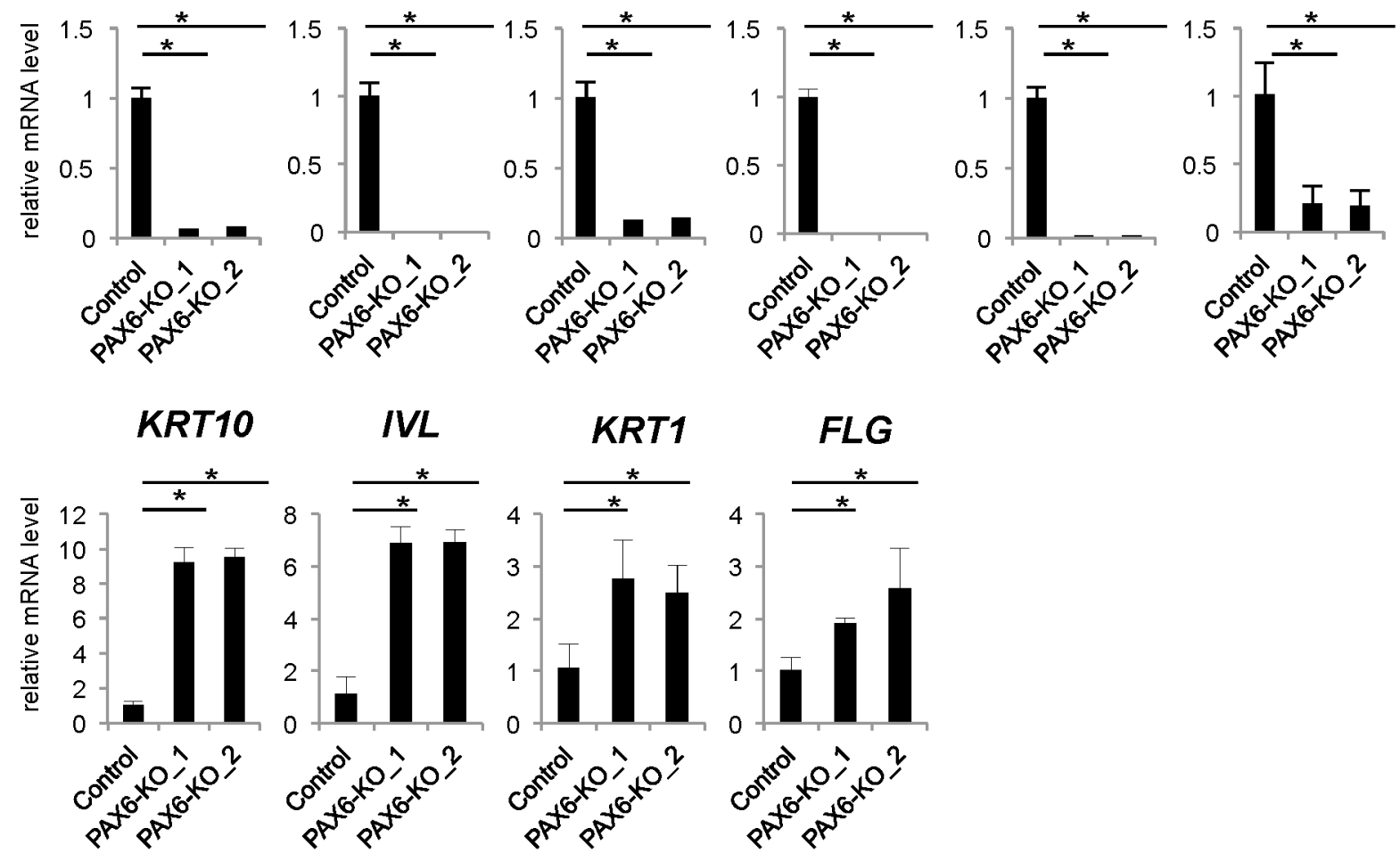

B

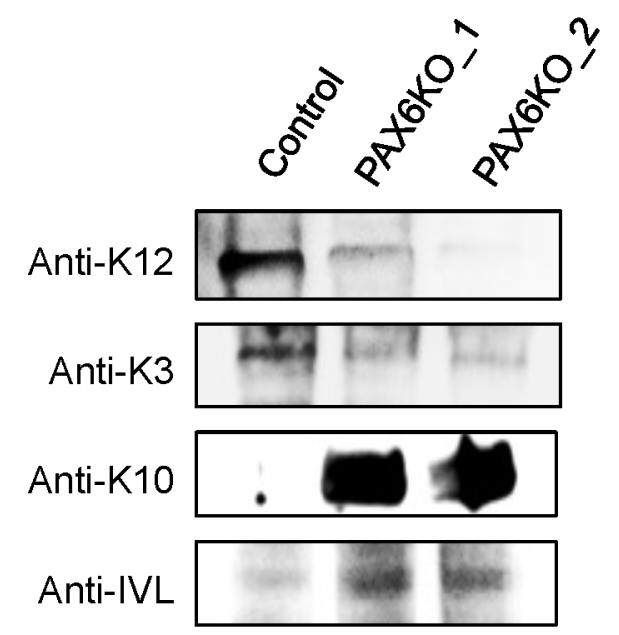

Anti-Histone-H3

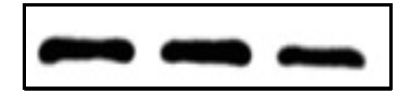


Corneal epithelial cells

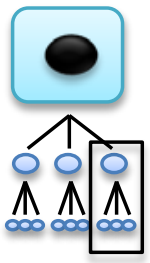

Corneal epithelium-related genes

- $\mathrm{K} 12, \mathrm{~K} 3$

- ALDH3A1

- CLU, TKT

- ANGPTL7

\section{Epidermal cells}

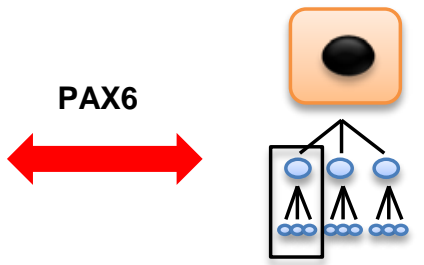

Epidermis-related genes

- K10, K1

- IVL

- FLG 